\title{
A Toolkit for Exploring Augmented Reality Through Construction with Children
}

\author{
Damien Brun \\ LICEF Research Center \\ Université TELUQ \\ Montréal, Canada \\ dbrun@licef.ca
}

\author{
Perrine Ruer \\ LICEF Research Center \\ Université TELUQ \\ Montréal, Canada \\ pruer@teluq.ca
}

\author{
Charles Gouin-Vallerand \\ LICEF Research Center \\ Université TELUQ \\ Montréal, Canada \\ cgouinva@teluq.ca
}

\author{
Sébastien George \\ UBL, Le Mans Université, \\ EA 4023, LIUM \\ 72085 Le Mans, France \\ sebastien.george@univ- \\ lemans.fr
}

\begin{abstract}
Augmented Reality begins to be widely mainstream among children, due to some interactive successes in video games and social networks. Based on this interest, we present CartonEd, an open and complete toolkit suitable for children dedicated to the construction of an augmented reality headset device. The toolkit let the children play and explore augmented reality with and beyond handheld devices. Inspired by the Do-It-Yourself movement, the toolkit includes different components such as blueprints, tutorials, videos, mobile apps, a software development kit and an official website. Among the mobile applications, one is implemented to guide the children through the construction process while experiencing augmented reality. To validate our solution (in particular the construction process and the guiding app) and understand its effect on children in regard to their relation to the augmented reality, we conducted four construction sessions. Our study examines the usability of the guiding app and the construction process. We report in this paper the main components of the CartonEd toolkit and the results of an evaluation among 57 children and teenagers (ages 8-16), showing a positive outcome about their own constructed device (all functional), their feelings and wishes regarding the augmented reality.
\end{abstract}

Keywords-augmented reality, construction, children, wearable, technology, do-it-yourself

\section{INTRODUCTION}

Nowadays mobile phones are the main medium to experience augmented reality. Mobile application such as video games (e.g. Pokemon Go [18]) and social media helped to make augmented reality widely available among the population. The main social networks added augmented reality face morphing features (filters) that are particularly used by the youth, allowing them to transform themselves into cats, unicorns or whatever offered by the platform. The potential of the augmented reality is not limited to those previous domains [3], for instance a lot of work has been done in education $[12,23]$, allowing students to interact and train their skill with an augmented reality book. Augmented reality for helping procedural tasks $[5,24]$ has also been subject to intensive research, consisting of adding contextual information during a specific process such as assembling. On the other hand, there is a proliferation of new hardware such as head-mounted display (HMD) [16] dedicated to augmented reality but due to the expensiveness and form factors of these kinds of devices, they are less available and hard to reach, in particular for young people, limiting their augmented reality experience to handheld devices.

Natural Sciences and Engineering Research Council of Canada
Therefore, we developed an affordable and open toolkit to make an augmented reality headset beneficial to children in many ways: (1) Anyone from young age is able to build the headset out of almost nothing (very simple materials). (2) Thanks to the openness, affordability and reachability of the toolkit, anyone is able and encouraged to use different kinds of materials and tools to change or customize the headset. (3) The construction process of a tangible augmented reality device generates both playful and pedagogical value, the technical simplicity of the headset makes the comprehension of augmented reality technology easier than using an industrial and commercial high-end headset.

The solution kit resulted from one and a half year of incremental, iterative and participatory design process, including previous experiments with adults, discussions with users and specialists of educational technology. The aim of this research through the complete toolkit and the study is to explore new augmented reality experience thanks to a functional inexpensive headset buildable by children.

The contribution of this paper is double. First, we report on the design and development of the toolkit main parts allowing the construction of an inexpensive augmented reality headset. Secondly, we report on a user test with 57 children, observing their own construction and analyzing the utilisability of an augmented reality guiding app. Finally, we provide everything freely from the project (guide, blueprint, mobile apps, software development kit, source code, etc.) to the teachers, designers and researchers to use it as an augmented reality educational technology.

\section{RELATED WORK}

Prior research projects explored low cost headset dedicated to augmented reality. Amer and Peralez [1] presented an affordable augmented reality device similar to the Google Cardboard (although this one is a virtual reality headset) for developing countries' population. Then, De Angeli and O'Neil [6] outlined their study in developing an augmented reality headset with low purchase and maintenance costs. The authors conducted a study to test the visual capacity of their tool depending on light condition of the environment and smartphone's screen brightness. Their results showed that using a smartphone could deliver very good rendering with ambient illumination similar to interior environment. However, none of the previous research projects offer a complete construction kit neither for adult, nor for children, ultimately greatly limiting access to this kind of augmented reality technology. Lately, Holokit.io [9] and Aryzon [2] two commercial products provided an inexpensive augmented reality headset made from cardboard but both of them include very specific mirror and lenses, making the kit not as easily available or constructible from 
nothing with simple material, and are not prone to customization, crafting and similar activities. They did not provide or suggest any user-test study about their augmented reality headset with children. Moreover, none of the previous solutions and project research introduced an augmented reality (or traditional) construction guide to their augmented reality headset.

On the one hand, a lot of studies from the maker movement have shown the benefit of a construction activity as an engaging educative and playful experience for children $[9,14,15,20]$. On the other hand, a lot of studies have explored augmented reality with children and shown a benefit in an educative context or not $[8,17,21,22]$. To create a toolkit engaging and enjoyable with potential educative benefits, our work borrow from both approaches: making and augmented reality with children. Thus, our work contributes a novel approach where the solution toolkit is beyond providing a highly affordable constructible augmented reality headset, it also adds enjoyable augmented reality experience along the construction process, which is a starting point for getting children interested in being more than a passive consumer of technology and give a better understanding of the augmented reality itself.

\section{A Constructing Augmented ReAlity TOOLKIT}

The following section presents our design and development of the toolkit, in particular the headset and the augmented reality guiding app.

\section{A. Concept}

The idea is to allow anyone and in particular children and teenagers to create an augmented reality headset device. To build the headset dedicated to augmented reality, the solution includes a guided construction app on a handheld device that also implement an augmented reality feature. Thus, the solution intertwines the construction process and its result from an augmented reality perspective, allowing users to experience and understand more deeply the impact and benefit of this kind of technology. It creates a link between the "how" and "what" of the headset around the augmented reality experience.

\section{B. Components}

The toolkit has many components, both hardware and software.

\section{1) Headset}

The headset (Fig. 1) is the tangible element of the toolkit following a Do-It-Yourself approach currently in its second version. The headset is made of simple materials with standard tools coupled with a smartphone, the complete list is presented in Fig. 2 (top). The use of a smartphone is primordial to reduce costs and most importantly to bring various essential components to the headset (chip, screen, camera, battery, connectivity, sensors). The second version of the toolkit is dedicated to children, there is no need to use potentially hazardous tools and materials such as utility knives and piece of sharp glass mirror. Then we made the blueprints with multiple geometrically simple shapes (as shown in the bottom of the Fig. 2) easier to understand instead of one single shape harder to cut and fold as in our first designed version made for adults [4].

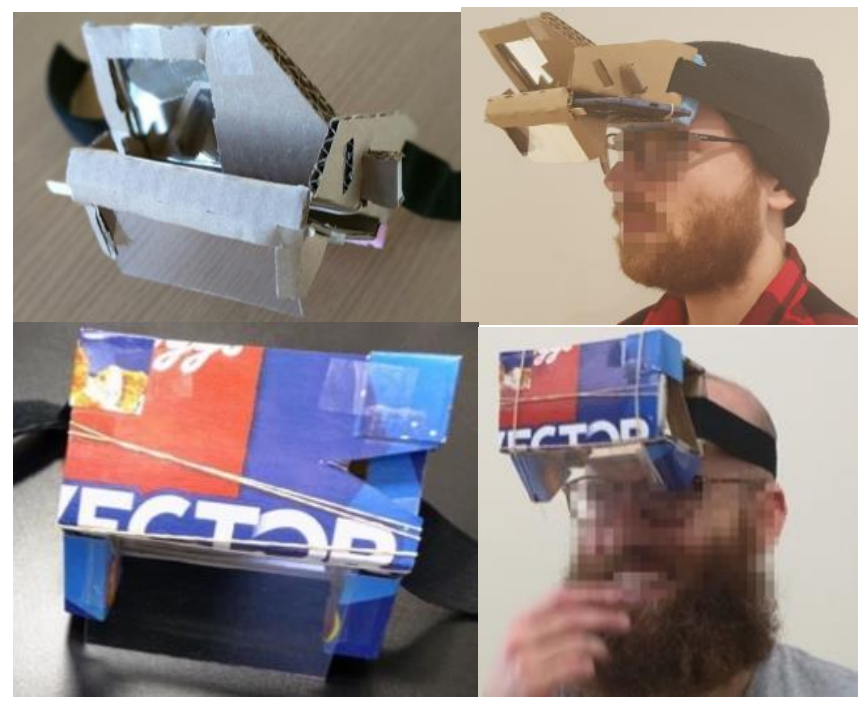

Fig. 1. Top: first version of the headset. Bottom: second version of the headset, easier to build with safer tools and material.

To construct the headset, the shapes from the blueprint presented in Fig. 2 (bottom) must be printed and paste to pieces of cardboard before being cut, folded and assembled altogether. Two kinds of cardboard are used, a few small parts from regular size thickness $(2 / 3 \mathrm{~mm})$ to solidify the headset, the rest is made from thin cardboard such as cereal box. A couple of other materials complete the headset, a sponge is placed between the headset and the user's forehead to make it more comfortable. A rubber band is added to secure the mobile phone and a utility stretch straps through the headset and around the head to hold it and allow handfree interaction. Finally, a piece of mirror (not glass but thin plastic) is positioned at $45^{\circ}$ to reflect the light of the mobile phone screen to the transparent plastic sheet (see Fig. 3), thus adding an artificial visual layer on the reality of the user. This visual configuration is an illusion technique called Pepper's ghost effect.

\section{2) Construction Guiding App}

The guiding app (shown in Fig. 4) is a mobile application dedicated to the construction of the headset. The aim of this app is to provide the instructions step by step supported by visual cues, an animation of a 3D model. As the user goes further into the different steps, the 3D model becomes a more complete headset. The animation at each step helps the user to understand how to fold each part and how to assemble them. The visual cue can be appreciated in two different ways: as a simple and regular 3D model presented on the handheld device (phone/tablet) that can be rotated with a finger gesture (swipe left or right), or as an augmented reality experience by aiming at a specific image (QR Code). The augmented reality feature respect the real size of the material and thus allow the user to easily compare their own construction with the virtual one. Along with the visual cue, each step includes a number, a short instruction and sometimes another advice all presented in Table 1. The first two steps (without number) are not related to the construction process but to explore the interaction of the guiding app (the first one dedicated to the swiping gesture and the second to the augmented reality feature). Two buttons allow the user to navigate through the different steps. 

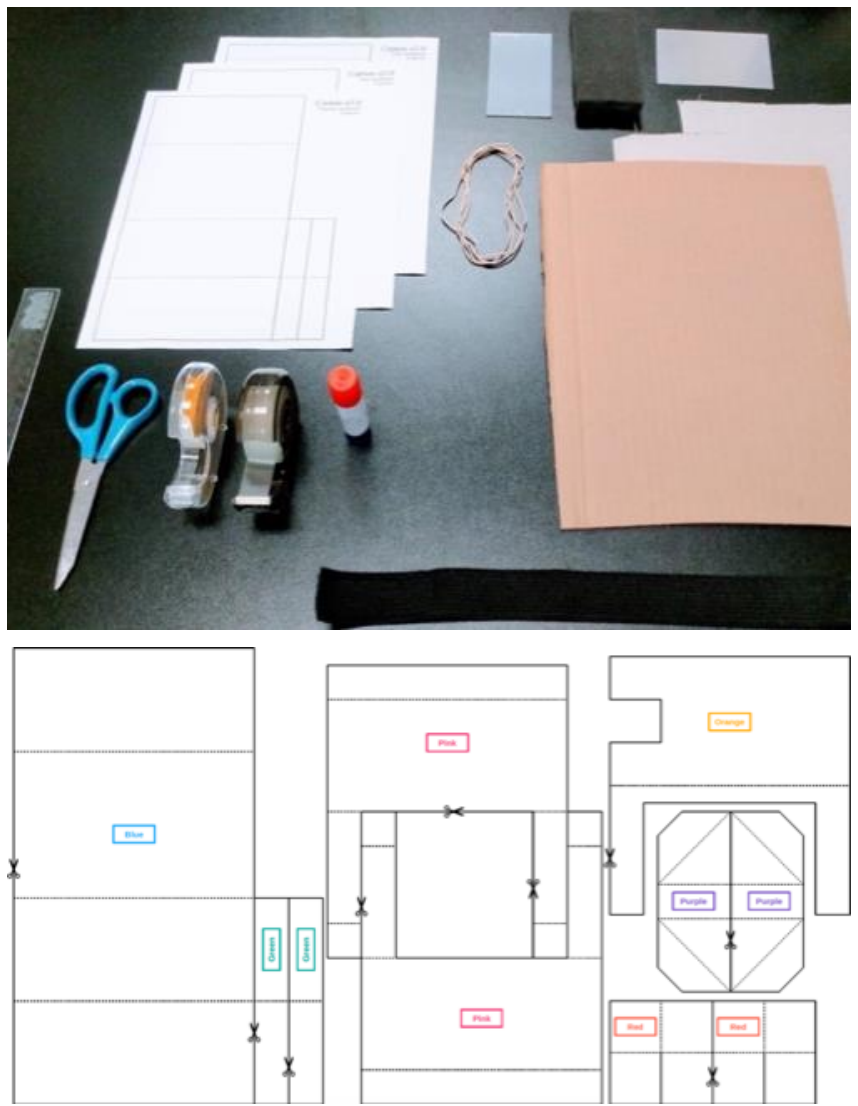

Fig. 2. Top: tools (rules, scissors, adhesive tape, glue stick), material (printed blueprints, cardboard, plastic mirror, transparent plastic sheet, sponge, rubber band and utility stretch straps). Bottom: blueprint of the headset, printable on regular paper format (US Letter / A4). They come with a simple legend (cut the continuous lines and fold the dotted lines).

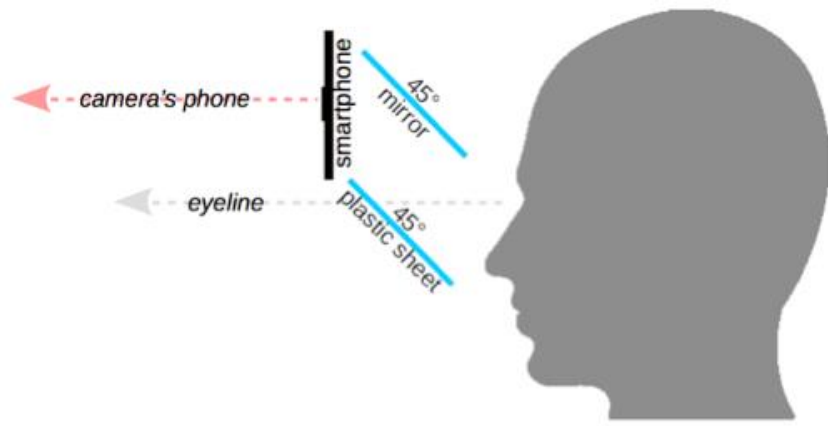

Fig. 3. Pepper's ghost effect configuration for the headset.

\section{3) Other Components}

The toolkit also includes other components which are briefly presented because they are not directly subject to the studies. Among them: a more traditional guiding tutorial dedicated to the construction available on Instructable, accompanied by videos of presentation and construction available on YouTube. We also developed an open source software development kit (SDK) for Android that allows developers to benefit from the full potential of the headset: for instance, by automatically set the screen parameters, or to recognize head movements (tilt and nod) for a hand-free interaction.
TABLE I. STEPS OF THE GUIDING APP

\begin{tabular}{|c|c|c|}
\hline $\mathbf{N}^{\circ}$ & Instruction & Advice \\
\hline \# & Observe the virtual headset & $\begin{array}{l}\text { Swipe left/right to rotate the } \\
\text { 3D models }\end{array}$ \\
\hline \# & Aim the QR Code on the paper & Turn around \\
\hline 1 & Check the tool list & You can share the tools \\
\hline 2 & Check the material list & Drag the list if needed \\
\hline 3 & Paste the paper on the cardboard & $\begin{array}{l}\text { Important to read the } \\
\text { blueprint legend }\end{array}$ \\
\hline 4 & Cut each continuous line & $\begin{array}{l}\text { Important to not cut the } \\
\text { dotted lines }\end{array}$ \\
\hline 5 & $\begin{array}{l}\text { After cutting, make sure to have } \\
\text { all } 10 \text { parts }\end{array}$ & \\
\hline 6 & $\begin{array}{l}\text { Fold the biggest regular } \\
\text { cardboard part (blue) }\end{array}$ & $\begin{array}{l}\text { Fold the dotted line, help } \\
\text { yourself with the rule and } \\
\text { adhesive }\end{array}$ \\
\hline 7 & $\begin{array}{l}\text { Paste the piece of mirror with } \\
\text { adhesive }\end{array}$ & $\begin{array}{l}\text { As flat as possible in the } \\
\text { center }\end{array}$ \\
\hline 8 & $\begin{array}{l}\text { Fold the two identical big parts of } \\
\text { thin cardboard (pink) }\end{array}$ & Fold the dotted line \\
\hline 9 & $\begin{array}{l}\text { Put together both previous parts } \\
\text { to form a structure }\end{array}$ & \\
\hline 10 & Fold the phone support (orange) & Fold the dotted line \\
\hline 11 & $\begin{array}{l}\text { Put together the mobile phone } \\
\text { support with the structure }\end{array}$ & Use adhesive tape \\
\hline 12 & $\begin{array}{l}\text { Add the sponge (opposite side of } \\
\text { the opening) }\end{array}$ & $\begin{array}{l}\text { Use adhesive tape on each } \\
\text { side }\end{array}$ \\
\hline 13 & $\begin{array}{l}\text { Insert the regular cardboard part } \\
\text { (blue) into the structure }\end{array}$ & Use adhesive tape \\
\hline 14 & $\begin{array}{l}\text { Fold the two identical small piece } \\
\text { of regular cardboard (green) }\end{array}$ & \\
\hline 15 & $\begin{array}{l}\text { Insert the two small pieces } \\
\text { (green) into the structure }\end{array}$ & Use adhesive tape \\
\hline 16 & $\begin{array}{l}\text { Fold the two identical purple } \\
\text { parts (thin cardboard) }\end{array}$ & Use adhesive tape \\
\hline 17 & $\begin{array}{l}\text { Put together the purple parts and } \\
\text { the structure }\end{array}$ & Use adhesive tape \\
\hline 18 & $\begin{array}{l}\text { Fold the two mobile phone } \\
\text { protection parts (red square) }\end{array}$ & Use adhesive tape \\
\hline 19 & Put together the protection parts & \\
\hline 20 & Add the transparent plastic sheet & $\begin{array}{l}\text { Do not bend the plastic } \\
\text { sheet }\end{array}$ \\
\hline 21 & $\begin{array}{l}\text { Insert the rubber band to make } \\
\text { sure the phone will not fall }\end{array}$ & $\begin{array}{l}\text { On each side around the } \\
\text { protection part }\end{array}$ \\
\hline 22 & Insert the utility stretch straps & $\begin{array}{l}\text { Must pass through the } \\
\text { regular cardboard part }\end{array}$ \\
\hline 23 & Finish! & Congratulations :- \\
\hline
\end{tabular}

The SDK is accompanied by documentation and two official demonstration apps which serve as samples all available on a GitHub repository. These open source apps are published on the Play Store allowing a user to quickly try its newly constructed headset. These apps include different features, such as an interactive tutorial to use the headset, a clock, an origami helper, a compass and a poster recognition providing additional visual information. Finally, an opensource dedicated website (https://mobicarton.github.io) allow anyone (in particular students, researchers, teachers, educators, developers and parents) to reach every component of the toolkit. 

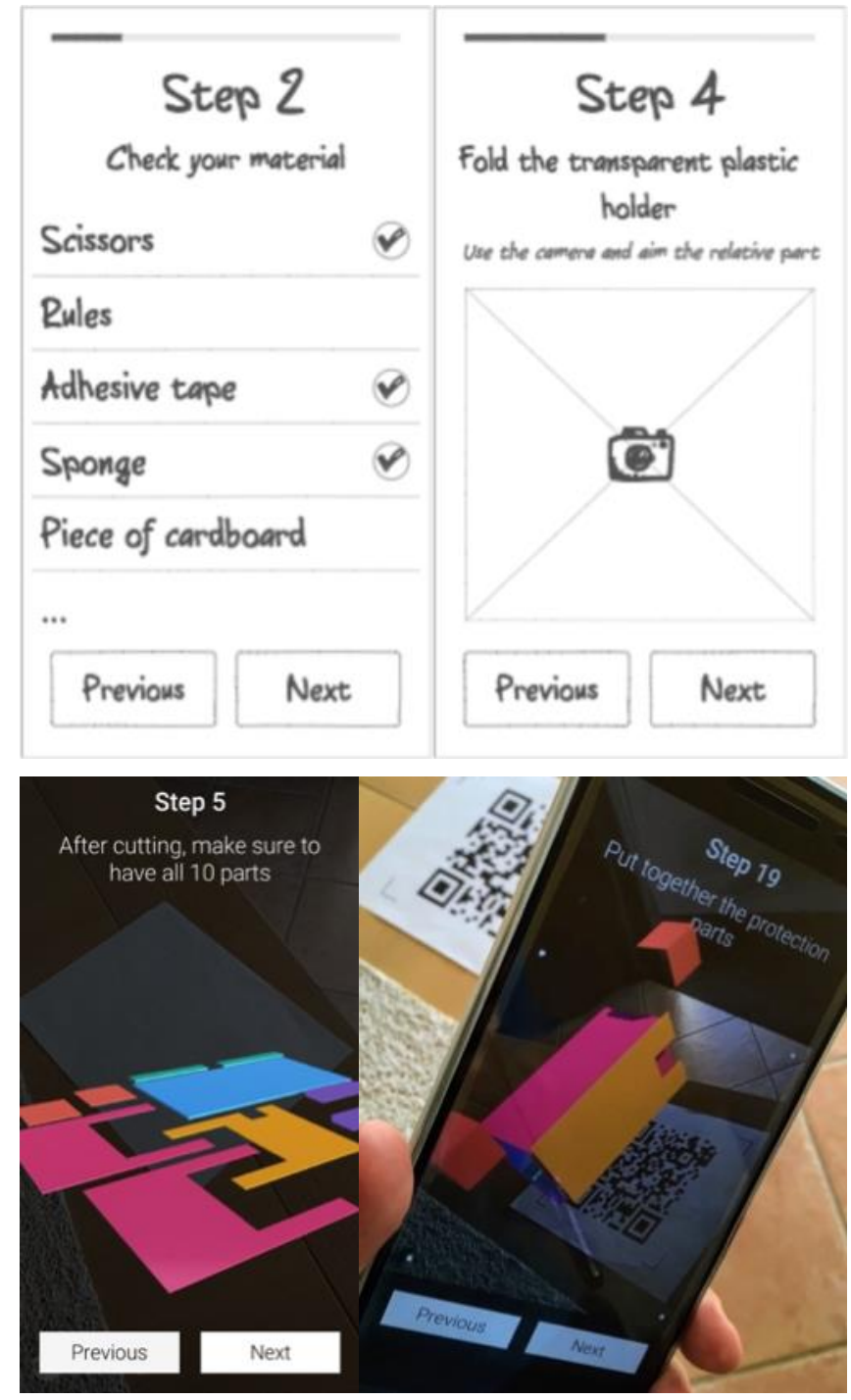

Fig. 4. Guiding app. From top left to bottom right: mock-up of the checklist, mock-up of the instruction step with the augmented feature, screenshot of a guiding app step without augmented reality (the model can be rotated with swipe gesture), picture of a guiding app step including augmented reality features.

\section{Implementation}

The headset's blueprints have been designed with Draw from OpenOffice. The guiding app was implemented using Unity3D. To recognize a specific image and to add 3D models above it, we used the augmented reality technology Vuforia. For the purpose of exploring the usability of the guiding app, we track some interactions (tap on buttons) and $\log$ them into a server using Firebase. Each interaction log contain the date, time, step and the state of the visual cue (augmented reality or not). The other components such as the SDK and demonstration apps were developed with Android Studio.

\section{EVALUATION}

We evaluated the solution, especially the headset and the guiding app first to ensure the constructability no matter the age, and second to understand the motivation and appreciation of the augmented reality technology with this toolkit among children. Hence we also compared their affinity between the different visual cue of the guiding app (augmented reality features or regular 3D models present on the screen of the handheld device).

\section{A. Pretest: Laboratory}

Following our iterative and participatory design process, before exploring the solution in the main study, we hosted an internal construction experiment with 2 children (a girl 9 and a boy 6 years old). They were asked (1) to build the headset with the help of the guiding app and (2) to answer a questionnaire. The main goal was to detect any flaws in the ongoing larger study and thus improve the blueprints, the headset, the construction process, the guiding app and the questionnaire. All the tools and materials were provided and the experiment lasted 2.5 hours. This pilot study point out the lack of visibility from the guiding app, it was hard to visually bond the virtual and the real headset currently constructed. Thus, we added color code to separate the different parts and emphasize on differentiation between them. This study also pointed out some misunderstanding with the instructions given by the guiding app, the lack of advices and a missing presentation of augmented reality in general. Based on these initial results (from the questionnaire and observation) we updated the toolkit, and also the main study presented in the next section.

\section{B. Main Study: Design and Recruiting}

The main study took place in a summer camp at a local school (metropolitan area). An explanation of the study (about the aim, the process, the data to collect and the research center) and a consent form were sent and returned signed by the participants and their parents. All these documents, as long as the study was approved by our Research Ethics Committee. On day one, participants were reminded that they can ask any questions, they are free to participate and could stop the experiment at any time without any penalty. Participants were 57 children between 8 and 16 years old $(M=11.7 ; S D=1.7)$, among them, 12 girls and 45 boys. We ran four different construction sessions. The main difference consists in the number of participants: 15 participants in the first session, then 10, 12 and 20 in the fourth and last session. Two researchers were present during each session, along with a team of organizers (3-4 young adults) from the school.

To compare the difference between the augmented reality (AR) feature and the regular (non-AR) visual cue of the guiding app, we forced the app to provide some steps in either one state (augmented reality or not). The augmented reality feature was available only from steps 14 to 23 (Table 1). Thus, each participant benefited from the two kinds of visual cues and could then express their preference, if any.

\section{Procedure}

For each session, the participants were divided (by themselves) in subgroups of 3 to 4 individuals around tables (Fig. 5). Each subgroup had to share some tools (scissors) and a provided mobile phone (Samsung S6) with the guiding app already installed. However all the materials were individually provided for each participant allowing them to create their own headset. The plastic mirror and transparent plastic sheet were already prepared (cut to the correct size). The researchers started all session by presenting themselves and gave a short overview of the study. It followed a brief 
presentation of augmented reality technology and its potential on everyday use ( 5 minutes), it was accompanied by Hyper-Reality (a concept film depicting a provocative vision of the future) from Keiichi Matsuda (6 minutes). Then, an introduction to the toolkit and its different components were given to the children ( 5 minutes), after that, the children started the construction process with the help of the guiding app (between 1 and 2 hours). The guiding app tracked each interaction and logged them to further postexperimental analyzes. During the construction process, researchers were instructed to intervene as little as possible. When the construction of the headset was finished, each participant tried their own headset with the official apps (clock, compass, origami tutorial, etc.) to check the integrity and functionalities of the headset. Finally, participants were told to answer a post-experiment questionnaire of 28 questions (presented in table 2) including both open and close questions. Some parts of the questionnaire were created to judge the usefulness and usability of the solution, inspired by the literature about ergonomic criteria for the evaluation of Human-Computer Interfaces [11]. At the beginning, demographic questions were asked, details about gender and age were collected as well as participants' usage of mobile phones to relate with skill and knowledge of technologies. Afterwards, participants were asked about the experiment, the construction process, their own construction (the headset) and the guiding app. Open questions inquired about how they would upgrade the headset or the experiment. For all the rated questions, the participants were asked to give a score between 1 (bad) and 5 (good). Despite having 28 questions, most of them are closed, therefore all the participants quickly fully filled in the questionnaire without much trouble. It took around 10 minutes for the participants to answer individually the printed questionnaire with the help of the researchers when needed. The same questionnaire was administered to all ages, few participants asked additional information or explanation to the questions. In total, each session lasted around 3 hours.

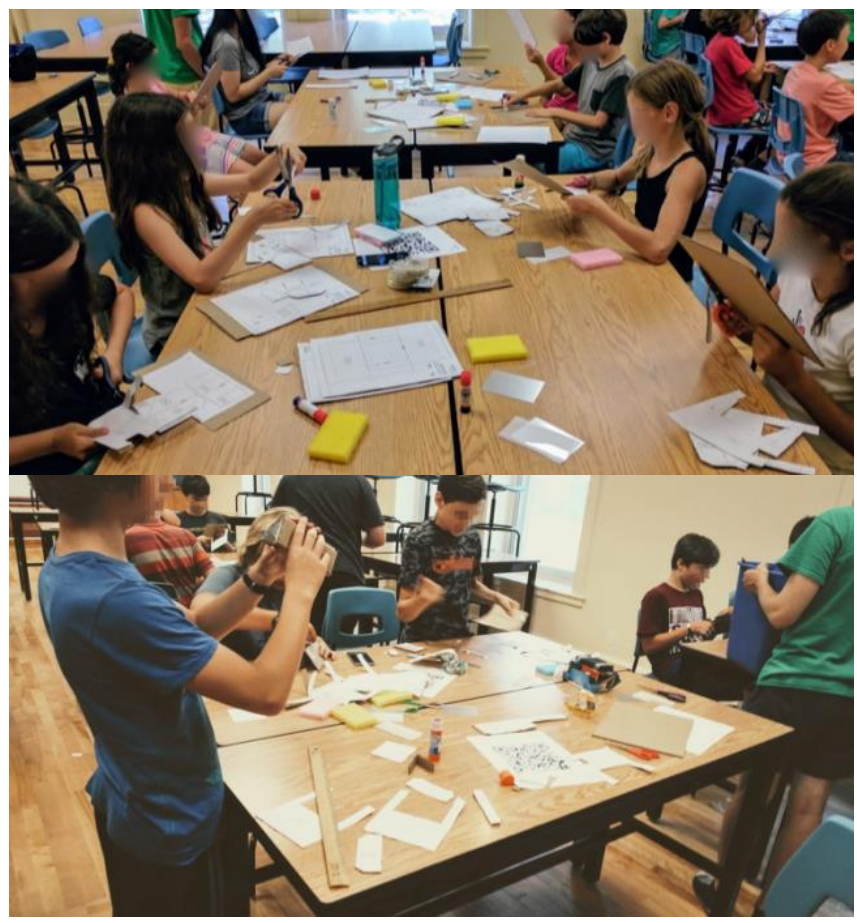

Fig. 5. Construction of the headset by children forming subgroups (16 in total from all sessions) around tables (sharing tools).
TABLE II. FULL SET OF QUESTIONS FROM THE QUESTIONNAIRE

\begin{tabular}{|c|c|c|}
\hline$\#$ & Questions & Answer type \\
\hline 1 & How old are you? & - \\
\hline 2 & Are you a girl or a boy? & Girl / Boy \\
\hline 3 & Do you use a smartphone? & $\begin{array}{l}\text { Every day / } \\
\text { Sometimes / Rarely / } \\
\text { Never }\end{array}$ \\
\hline 4 & Are you satisfied with your creation? & $\begin{array}{l}1 \text { (unsatisfied) to } 5 \\
\text { (satisfied) }\end{array}$ \\
\hline 5 & Was it easy to build? & $\begin{array}{l}1 \text { (hardly) to } 5 \\
\text { (easily) }\end{array}$ \\
\hline 6 & $\begin{array}{l}\text { The allocated time was enough to build } \\
\text { the device? }\end{array}$ & $\begin{array}{l}1 \text { (not enough) to } 5 \\
\text { (ideal) }\end{array}$ \\
\hline 7 & $\begin{array}{l}\text { Are you proud to use a product you } \\
\text { build yourself? }\end{array}$ & Yes / No \\
\hline 8 & $\begin{array}{l}\text { Would you like to update and customize } \\
\text { your creation? }\end{array}$ & Yes / No \\
\hline 9 & If yes, what would you like to do? & Open \\
\hline 10 & $\begin{array}{l}\text { Do you like the openness of the project? } \\
\text { (It means that the blueprints, guides, etc. } \\
\text { are available for everyone) }\end{array}$ & Yes / No \\
\hline 11 & $\begin{array}{l}\text { Did you know the concept of augmented } \\
\text { reality before this experiment? }\end{array}$ & Yes / No \\
\hline 12 & If yes, from which application? & Open \\
\hline 13 & $\begin{array}{l}\text { Do you know the difference between } \\
\text { augmented reality and virtual reality? }\end{array}$ & Yes / No \\
\hline 14 & If yes, could you explain it? & Open \\
\hline 15 & $\begin{array}{l}\text { Do you like the augmented reality and } \\
\text { its potential? }\end{array}$ & Yes / No \\
\hline 16 & $\begin{array}{l}\text { Di you have pleasure to use the mobile } \\
\text { app to guide the construction? }\end{array}$ & $\begin{array}{l}\text { A lot / Quite / Not } \\
\text { enough / None }\end{array}$ \\
\hline 17 & $\begin{array}{l}\text { Could you score different characteristics } \\
\text { (design, interactions, contents) of the } \\
\text { mobile app? }\end{array}$ & 1 (bad) to 5 (good) \\
\hline 18 & $\begin{array}{l}\text { Was it easy to use the camera to show } \\
\text { the 3D contents? }\end{array}$ & $\begin{array}{l}1 \text { (hardly) to } 5 \\
\text { (easily) }\end{array}$ \\
\hline 19 & $\begin{array}{l}\text { Do you prefer having the } 3 \mathrm{D} \text { contents in } \\
\text { AR or in its regular form? }\end{array}$ & $\begin{array}{l}\text { AR / Regular form / } \\
\text { Equally }\end{array}$ \\
\hline 20 & $\begin{array}{l}\text { Would you like to have more } \\
\text { "traditionnal" courses with AR? (Such } \\
\text { as geography, physics, mathematics, } \\
\text { literature...) }\end{array}$ & Yes / No \\
\hline 21 & $\begin{array}{l}\text { Would you like to have a similar mobile } \\
\text { app as a course support? (For instance, } \\
\text { to use at home) }\end{array}$ & Yes / No \\
\hline 22 & $\begin{array}{l}\text { Does the experiment make you excited } \\
\text { about augmented reality and more } \\
\text { generally new technologies? }\end{array}$ & $\begin{array}{l}\text { Yes / No / I do not } \\
\text { know }\end{array}$ \\
\hline 23 & $\begin{array}{l}\text { Does the mobile application motivate } \\
\text { you to use AR to build other products? }\end{array}$ & Yes / No \\
\hline 24 & $\begin{array}{l}\text { Does the fact that building the device } \\
\text { with other children around motivate } \\
\text { you? }\end{array}$ & Yes / No \\
\hline 25 & $\begin{array}{l}\text { Would you recommend to someone of } \\
\text { your family or friends to use the mobile } \\
\text { app to build their own device? }\end{array}$ & $\begin{array}{l}\text { Sure / Probably / Not } \\
\text { sure / Not at all }\end{array}$ \\
\hline 26 & Did you ask some help to build it? & No / Once / Few \\
\hline 27 & How did you appreciate the experiment? & $\begin{array}{l}1 \text { (not at all) to } 5 \\
\text { (super) }\end{array}$ \\
\hline 28 & $\begin{array}{l}\text { Do you have any suggestion about the } \\
\text { experiment or the questionnaire? }\end{array}$ & Open \\
\hline
\end{tabular}

\section{RESULTS}

Results come from the questionnaires, the researchers' observations and also from the logs of the guiding app. The results are organized as such: (1) assessing the experience and apprehension of augmented reality from the participants (2) reporting on the constructability of the headset (3) 
highlighting difference analysis based on age and previous experience (4) assessing the effect of the experiment on the perception of augmented reality.

\section{A. Augmented Reality and Technology in General}

Over 57 children, less than 10 per cent $(9 \%)$ never used a mobile phone before the experiment. Others, $61 \%$ own one mobile phone and use it every day, the rest has already used a mobile phone at least once from a family member or friend. Around $60 \%$ already knew or at least heard about the concept of augmented reality before participating in the study, most of them from video games, in particular Pokemon GO $(n=11)$. To be sure that participants understood the concept of augmented reality, we ask them to define it and point its differences with virtual reality: $63 \%$ felt confident to give an answer (mostly correct). Almost all the participants, $95 \%$ liked the potential of augmented reality.

\section{1) Questionnaire}

Most of the participant, $95 \%$ had a pleasure to use the construction guiding app. However, 5\% did not have enough pleasure (explicit reasons unknown). The design, interaction and contents of the guiding app were all individually scored at $4.0 / 5$ on average $(S D=1.1)$.

Half of the participants had no preferences between the augmented reality over the non-augmented reality visual cue presented in the guiding app. Then, a quarter preferred the augmented reality visual cue and the last quarter the nonaugmented reality visual cue. The easiness of targeting a QR Code to bring up the $3 \mathrm{D}$ model was rated at $3.6 / 5$ on average $(S D=1.2)$.

A majority of the participant, $75 \%$ answered that they would appreciate using this kind of guiding app also to other construction projects. Even, $63 \%$ would also like to have an augmented reality course with educative oriented content such as history, geography, physics, chemistry, mathematics and literature. More of them, $84 \%$ would appreciate having a similar simple guiding app with instruction about a specific course as a support to be used at home or in the classroom.

\section{2) $\log s$}

We tracked 4431 interaction from the two buttons. 2558 $(57.7 \%)$ taps on the "next" button and the rest (1873) on "previous". Which denotes many back and forth in the steps. Also, 69\% (11/16) of the subgroups started by quickly exploring the construction process and went first through all the different steps.

\section{B. Construction of the Headset}

\section{1) Questionnaire}

The satisfaction of their own construction scored on average at $3.8 / 5(S D=1.0)$ and $91 \%$ were proud to use a device they constructed themselves. The participants scored the easiness of construction at 3.3 (1=hard; 5=easy) on average $(S D=1.1)$ with an allocated time judged at 3.6 ( $1=$ inadequate; $5=$ adequate) on average $(S D=1.1)$. All of them made a functional headset. However, the quality differs, depending on their personal skills, for instance a participant (10 years old) who performed very well in the construction of his headset told the researchers that he made a lot of origami, which shares similar needed skills (manual, geometrical and spatial). Due to the controlled experiment settings, they had minimal required materials and tools, their customization has been limited. Therefore, $58 \%$ wanted to customize the headset: "decorating/coloring" $(n=10)$ and "make it compatible with the iPhone" $(n=3)$ were the most common things they wanted to do. The second customization was not related to the headset itself, but to the software part. Despite being told to intervene as little as possible, the researchers and organizers had to sometimes help the children during the construction process, mainly for the step 11 (putting together the mobile phone holder with the structure) because the visual cue and the given instruction was not accurate enough.

\section{2) $\log s$}

From the logs, the step with the longest duration was the number 4 (cutting each shape from the cardboard) with a means of 9 minutes and 36 seconds and is followed by the step 3 (paste the paper on the cardboard) with 8 minutes and 13 seconds on average. The next longest steps are the 11,12 and 13, all just above 5 minutes. These durations correlate with the children asking some help because of the difficulty of step 11 and then figuring out sometimes 1 or 2 steps after. Most of the other steps lasted around 3 minutes.

\section{Difference Analysis}

This part presents an analysis difference based on the age of the participants and their experience to augmented reality. Answers to the scale (1 to 5) questions are reported in graphs below.

\section{1) Age}

We removed the age 8, 15 and 16 years old from the difference analysis because they only had respectively 1,2 and 1 participants. As shown in Fig. 6 (top and bottom), most of the answers increased slightly for 10 years old. Only the question 4, 6, and 18 (bottom of the Fig. 6) show significant variation (above 1.0). The questions 6 and 18 follow the same pattern, starting at $3.0 / 5$ to 9 years old and increasing until 4.0/5 and above at 14 years old, which could be due to an increase of skills along with the age, thus the time allocated is considered enough and the manipulation of the mobile phone to aim the QR Code with the camera is getting better as the participant is getting older.

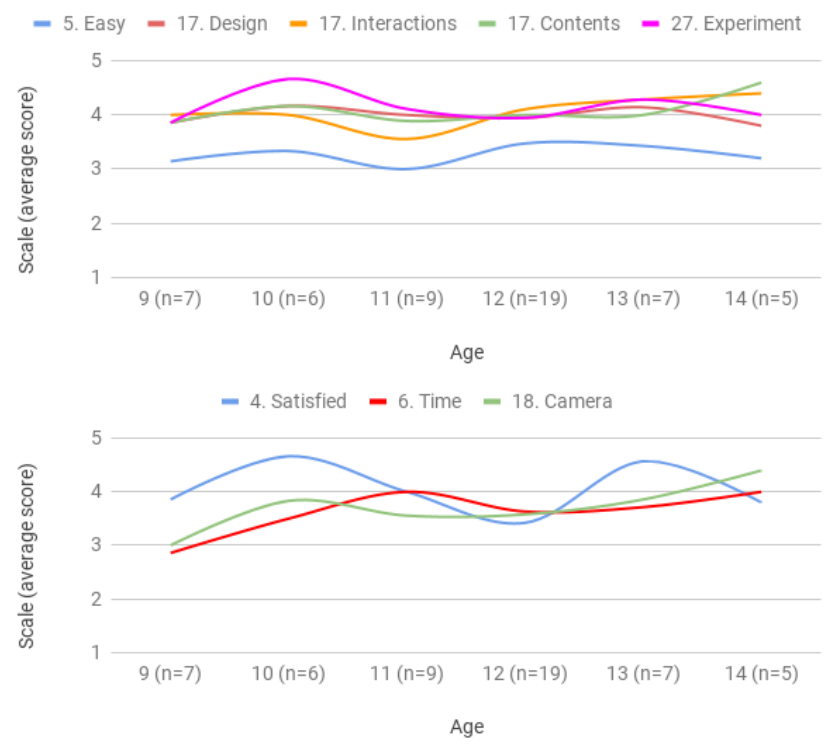

Fig. 6. Difference analysis based on scale questions according to participants' age. Top: questions with low variation to the score (around 0.5). Bottom: questions with significant variation to the score (superior to $1.0)$. 


\section{2) Experience}

Question 11 allows us to differentiate participants with previous experience to augmented reality $(n=34)$ from participants that did not know the concept of augmented reality before the experiment $(n=23)$. Interestingly Fig. 7 shows that most of the score a slightly better for participants that did not have previous experience to augmented reality, in particular the satisfaction of their creation (Q. 4) and the experiment in general (Q. 27), probably due to personal excitation of getting into a totally new technology. We also found similar results from the other closed questions (such as "yes/no").

\section{Experimentation in General}

To the question if they felt more motivated and engaged in augmented reality and more generally with technology after the experiment, $44 \%$ answered yes, $46 \%$ answered they did not know and $10 \%$ answered no. Finally, the experiments as a whole (including presentation, video and construction) was greatly appreciated by the participants $(M=4.1 / 5$, $S D=0.8$ ).

\section{DISCUSSION, LIMITATIONS AND FUTURE WORK}

The results showed us that the toolkit is appropriate to children and teenagers. They were able to follow the augmented reality guide and build their own augmented reality device in addition to test it and enjoy the whole experiment of exploring and experiencing augmented reality. We think teachers and educators can use this project to at least introduce the augmented reality technology to children, in particular the neophyte.

However, minor struggle to reveal the augmented reality 3D model with the QR Code could have slightly decreased their appreciation of augmented reality. We have since implemented a marker-less augmented reality technology that could alleviate this problem. We also noticed that participants in the study were not always constructing at the same speed even those in the same subgroup of children sharing only one mobile phone, hence the same guiding app. This caused some undesired and accented back and forth through the steps which limited the utilization of the logs. To improve the study and application of the toolkit, limiting one mobile phone per participant would solve this problem.

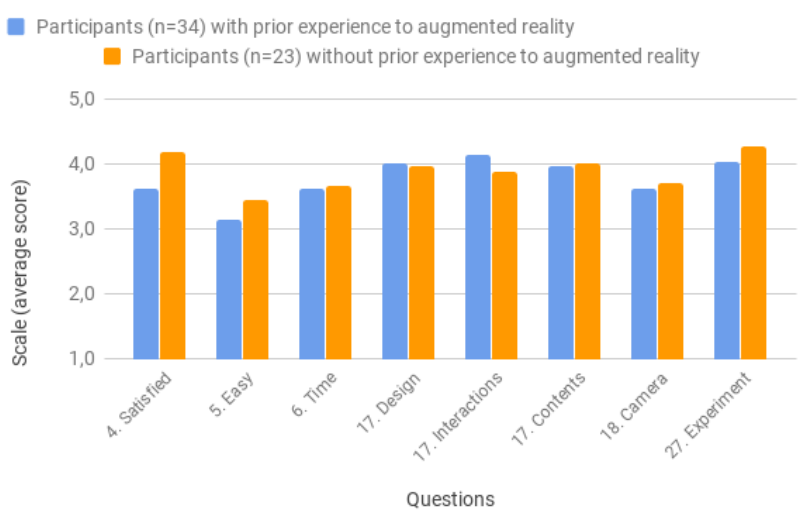

Fig. 7. Difference analysis based on scale (1 to 5) questions according to participants' experience with augmented reality.
In our study, the comparison between regular and augmented reality visual cues is limited to some specific steps only, that could have a side effect on the appreciation (positive or negative). A rotation of the step dedicated to augmented reality regarding each group could be done to address this limitation. Also, as an improvement, the toolkit already includes the use of a food container to the construction but we are in the process of including more waste material (such as transparent plastic bottle) improving the recycling and creativity process of the children. Interestingly, we did not assess the use of the headset itself because we emphasized on the construction process, however, thanks to observations, we assure that the children really enjoyed using it and they continued to use and test the headset by themselves with different mobile apps already installed on the phone, such as YouTube and some of them expressed the willingness to create their own app. Therefore, we will explore the creation of augmented reality content dedicated to this kind of headset with children by using for instance Scratch [6, 22]. Finally, base on the solid groundwork of the presented solution, we will and encourage others to adapt the toolkit to further domains (even away from technology) such as science. For instance, dedicated to an optic course, the visual effects and concepts of light reflection, distortion, stereoscopy and Pepper's ghost effect could be explored and learned along with the construction of the headset and thus benefiting from the motivation and enthusiast of the complete augmented reality construction toolkit.

\section{CONCLUSION}

In this work we presented CartonEd, a new toolkit for constructing an augmented reality headset with the help of the augmented reality itself. We reported on the design of the toolkit, in particular the construction process and the guiding mobile app and on an evaluation of the solution among 57 children. We found that children greatly engaged with the augmented reality guiding app, which allowed them to build a functional augmented reality headset from simple material and tools, no matter how old they were. Children who did not experience augmented reality before the experiment tended to slightly prefer the solution. However, during the study all the children have shown a great interest in the augmented reality technology more generally and are eager to experience it in different contexts for educational purpose.

Finally, we believe our toolkit to be useful for the teachers, educators, designers, researchers and developers that want to integrate the solution into different context: that could be into the classroom or at home to introduce augmented reality such as the presented experiment or to go further into another domain such as studying physics through the construction of an augmented reality headset for children. The solution including the experiment could also be adapted and used by various companies for team building purpose or as a marketing asset offering customizable and highly interactive experience. In this regard, we made the toolkit fully available and open to everyone. 


\section{ACKNOWLEDGMENT}

We would like to thank all the participants (children and organizer of the summer camp from Collège Durocher SaintLambert) and all the publication support and staff, who wrote and provided helpful comments on previous version of this document. As well authors gratefully acknowledge the grant from Natural Sciences and Engineering Research Council (NSERC) of Canada and the company Black Artick.

\section{REFERENCES}

[1] Ahmed Amer and Phillip Peralez. "Affordable Altered Perspectives: Making Augmented and Virtual Reality Technology Accessible." In 2014 IEEE Global Humanitarian Technology Conference (GHTC), 603-8, 2014.

[2] "Augmented Reality powered by your smartphone - Aryzon" Accessed June 20, 2018. https://aryzon.com/.

[3] Billinghurst, Mark, Adrian Clark, and Gun Lee. "A Survey of Augmented Reality." Interaction 8, no. 2-3 (2014): 73-272.

[4] Damien Brun, Susan M. Ferreira, Charles Gouin-Vallerand, Sébastien George, (2017) "A mobile platform for controlling and interacting with a do-it-yourself smart eyewear", International Journal of Pervasive Computing and Communication, Vol. 13 Issue 1, pp.41-61.

[5] Sebastian Büttner, Henrik Mucha, Markus Funk, Thomas Kosch, Mario Aehnelt, Sebastian Robert, and Carsten Röcker. "The Design Space of Augmented and Virtual Reality Applications for Assistive Environments in Manufacturing: A Visual Approach." In Proceedings of the 10th International Conference on PErvasive Technologies Related to Assistive Environments, 433-440. ACM, 2017.

[6] Sayamindu Dasgupta and Benjamin Mako Hill. "Scratch Community Blocks: Supporting Children As Data Scientists." In Proceedings of the 2017 CHI Conference on Human Factors in Computing Systems, 3620-3631. CHI '17. New York, NY, USA: ACM, 2017.

[7] Daniela De Angeli and Eamonn J. O'Neill. "Development of an Inexpensive Augmented Reality (AR) Headset." In Proceedings of the 33rd Annual ACM Conference Extended Abstracts on Human Factors in Computing Systems, 971-976. CHI EA '15. New York, NY, USA: ACM, 2015.

[8] Stéphanie Fleck, Martin Hachet, and J. M. Christian Bastien. "Marker-Based Augmented Reality: Instructional-Design to Improve Children Interactions with Astronomical Concepts." In Proceedings of the 14th International Conference on Interaction Design and Children, 21-28. IDC '15. New York, NY, USA: ACM, 2015.

[9] Erica Rosenfeld Halverson, and Kimberly Sheridan. "The Maker Movement in Education." Harvard Educational Review 84, no. 4 (2014): 495-504.

[10] "HoloKit: Open Source Mixed Reality for Everyone." Accessed June 20, 2018. https://holokit.io/

[11] Bastien, JM Christian, and Dominique L. Scapin. "Ergonomic criteria for the evaluation of human-computer interfaces". Diss. Inria, 1993.
[12] Kangdon Lee. "Augmented Reality in Education and Training." TechTrends 56, no. 2 (2012): 13.

[13] Priscilla Little, Christopher Wimer, Heather B. Weiss, and others. "After School Programs in the 21st Century: Their Potential and What It Takes to Achieve It." Issues and Opportunities in out-of-School Time Evaluation 10, no. 1-12 (2008).

[14] Lee Martin. "The Promise of the Maker Movement for Education." Journal of Pre-College Engineering Education Research (J-PEER) 5, no. 1 (April 29, 2015).

[15] Lee Martin, and Colin Dixon. "Youth Conceptions of Making and the Maker Movement." In Interaction Design and Children Conference, New York, 2013.

[16] Paul Milgram, Haruo Takemura, Akira Utsumi, Fumio Kishino, and others. "Augmented Reality: A Class of Displays on the RealityVirtuality Continuum." In Telemanipulator and Telepresence Technologies, 2351:282-292, 1994.

[17] Sascha Oberhuber, Tina Kothe, Stefan Schneegass, and Florian Alt. "Augmented Games: Exploring Design Opportunities in AR Settings With Children." In Proceedings of the 2017 Conference on Interaction Design and Children, 371-377. IDC '17. New York, NY, USA: ACM, 2017.

[18] Janne Paavilainen, Hannu Korhonen, Kati Alha, Jaakko Stenros, Elina Koskinen, and Frans Mayra. "The PokéMon GO Experience: A Location-Based Augmented Reality Mobile Game Goes Mainstream." In Proceedings of the 2017 CHI Conference on Human Factors in Computing Systems, 2493-2498. CHI '17. New York, NY, USA: ACM, 2017.

[19] Patrice Potvin, and Abdelkrim Hasni. "Interest, Motivation and Attitude towards Science and Technology at K-12 Levels: A Systematic Review of 12 Years of Educational Research." Studies in Science Education 50, no. 1 (January 2, 2014): 85-129.

[20] Sandra Schön, Martin Ebner, and Swapna Kumar. "The Maker Movement. Implications of New Digital Gadgets, Fabrication Tools and Spaces for Creative Learning and Teaching." ELearning Papers 39 (2014): 14-25.

[21] Radu, Iulian. "Augmented Reality in Education: A Meta-Review and Cross-Media Analysis." Personal Ubiquitous Comput. 18, no. 6 (August 2014): 1533-1543.

[22] Iulian Radu and Blair MacIntyre. "Augmented-Reality Scratch: A Children's Authoring Environment for Augmented-Reality Experiences." In Proceedings of the 8th International Conference on Interaction Design and Children, 210-213. IDC '09. New York, NY, USA: ACM, 2009.

[23] Marc Ericson C. Santos, Angie Chen, Takafumi Taketomi, Goshiro Yamamoto, Jun Miyazaki, and Hirokazu Kato. "Augmented Reality Learning Experiences: Survey of Prototype Design and Evaluation." IEEE Transactions on Learning Technologies 7, no. 1 (2014): 38-56.

[24] Arthur Tang, Charles Owen, Frank Biocca, and Weimin Mou. "Comparative Effectiveness of Augmented Reality in Object Assembly." In Proceedings of the SIGCHI Conference on Human Factors in Computing Systems, 73-80. ACM, 2003. 